\title{
"Lattice-Free" Simulations of Topological Defect Formation
}

\author{
Robert J. Scherrer, \\ NASA/Fermilab Astrophysics Center, \\ Fermi National Accelerator Laboratory, \\ P.O. Box 500, \\ Batavia, IL 60510, \\ and \\ Department of Physics and Department of Astronomy, \\ The Ohio State University, \\ Columbus, Ohio 43210 \\ Alexander Vilenkin \\ Institute of Cosmology, Department of Physics and Astronomy, \\ Tufts University, Medford, MA 02155
}

\begin{abstract}
We examine simulations of the formation of domain walls, cosmic strings, and monopoles on a cubic lattice, in which the topological defects are assumed to lie at the zeros of a piecewise constant 1, 2, or 3 component Gaussian random field, respectively. We derive analytic expressions for the corresponding topological defect densities in the continuum limit and show that they fail to agree with simulation results, even when the fields are smoothed on small scales to eliminate lattice effects. We demonstrate that this discrepancy, which is related to a classic geometric fallacy, is due to the anisotropy of the cubic lattice, which cannot be eliminated by smoothing. This problem can be resolved by linearly interpolating the field values on the lattice, which gives results in good agreement with the continuum predictions. We use this procedure to obtain a lattice-free estimate (for Gaussian smoothing) of the fraction of the total length of string in the form of infinite strings: $f_{\infty}=0.716 \pm 0.015$.
\end{abstract}

\section{INTRODUCTION}

Topological defects arise when the manifold of the degenerate vacuum states of a field is not simply connected. (See Ref. [1] for a review). In the simplest set of models, domain walls arise from a one-component field with two degenerate vacuum states, cosmic strings correspond to a two-component field in which the degerate vacua form a circle in the field space, and monopoles arise from a three-component field for which the degenerate vacua lie on the surface of a sphere.

The formation of topological defects can be simulated by laying down the appropriate field on a cubic lattice, and identifying the corresponding topological defects with zeros of the field. Although the most effort has gone toward simulations of cosmic string formation [2] - [9], similar investigations have also been undertaken for the study of domain walls [10] - 12] and monopoles [13] - 14].

In this paper, we point out a potential problem for some such lattice-based simulations. Even when the fields which give rise to the defects are smoothed on a scale larger than the lattice spacing (as in Ref. [8]), residual lattice effects remain. These are due to the fact that a continuous surface cannot be distorted to lie on the edges of a lattice without also distorting the area of the surface.

In the next section, we discuss our topological defect simulations, and we provide analytic expressions for the density of walls, strings, and monopoles. These analytic predictions are compared with the results of our numerical simulations, and they are found to disagree. In Sec. 3, a second set of analytic predictions, which does agree with the simulations, is presented, and we explain the origin of the discrepancy, which is closely related to a classic geometric fallacy. A second set of simulations using linear interpolation of the fields is presented, and these are shown to agree with analytic continuum predictions. The implications are discussed in Sec. 4. Our interpolation scheme produces a lattice-free estimate for the fraction of string length in infinite strings (with Gaussian smoothing): $f_{\infty}=0.716 \pm 0.015$. 


\section{THE PROBLEM}

In an earlier paper [8] we simulated the formation of cosmic strings from correlated fields on a cubic lattice, smoothing the field on small scales to eliminate lattice effects. Here we extend these simulations to domain walls and monopoles.

Consider first the case of domain walls. We can assign a real scalar field $\phi$ to each of the cells of a cubic lattice, and the location of the domain wall is then identified with faces of the lattice across which the value of the field crosses zero. To simulate cosmic strings rather than domain walls, we use a complex scalar field, or equivalently, a two-component real field $\phi$. Then the zeros of $\phi$ will lie on the edges of the lattice, which we then identify as the location of the strings. Finally, to simulate monopoles, we take $\phi$ to be a three-component real field; the zeros of $\phi$ then form a set of disconnected points lying on the vertices of the lattice; this gives the location of the corresponding monopoles.

Following the procedure in Ref. [8], we take $\phi$ to be a Gaussian random field. Then we can exploit the fact that the components of an $N$-component Gaussian field, $\phi_{1}, \phi_{2}, \ldots \phi_{N}$, are themselves independent real Gaussian fields. Hence, our model for cosmic strings is equivalent to using two independent real Gaussian fields (which can be taken to be $\phi_{1}$ and $\phi_{2}$ ). The zeros of these two fields form two independent sets of surfaces, and the strings lie at the intersection of these surfaces. Similarly, our field configuration for monopoles can be treated as three independent real Gaussian fields, $\phi_{1}, \phi_{2}$, and $\phi_{3}$. The zeros of these three fields form three sets of surfaces, and their intersection is a set of disconnected points, giving the location of the monopoles.

A Gaussian field is completely determined by its power spectrum $P(k)$, defined by

$$
P(k)=\int d^{3} r e^{i \mathbf{k} \cdot \mathbf{r}}\langle\phi(\mathbf{x}) \cdot \phi(\mathbf{x}+\mathbf{r})\rangle .
$$

Following the procedure in Ref. [8], we assume a power-law power spectrum of the form

$$
P(k) \propto k^{n} .
$$

Note that this is equivalent to taking each of the components $\phi_{i}$ to have a power spectrum given by equation (2). We add a backround non-zero mean field to the box to simulate the effect of long-wavelength modes (see Ref. [8] for the details). We also smooth the field $\phi$ on small scales with a Gaussian window function

$$
W(r)=\exp \left(-r^{2} / 2 r_{0}^{2}\right)
$$

where the smoothed field $\phi_{s}(\mathbf{x})$ is the convolution of $\phi(\mathbf{x})$ with $W(\mathbf{r})$ :

$$
\phi_{s}(\mathbf{x})=\int d^{3} r \phi(\mathbf{x}+\mathbf{r}) W(\mathbf{r})
$$

This Gaussian smoothing changes the power spectrum from $P(k)$ to $P_{s}(k)$, given by

$$
P_{s}(k)=P(k) e^{-k^{2} r_{0}^{2}}
$$

(Again, equation (4) is equivalent to smoothing each of the components $\phi_{i}$ with $W(\mathbf{r})$, and equation (5) can similarly be applied separately to the power spectrum for each component.) The effect of smoothing is to reduce the magnitude of the small-scale fluctuations by averaging them out over the window function. One might hope that if $r_{0}$ is taken to be larger than the lattice spacing, lattice effects can be reduced or eliminated entirely. We will see that this is not the case.

Given this model, it is possible to calculate the topological defect density analytically. Our starting point is a set of results due to Ryden [15] concerning the properties of level-crossing surfaces in Gaussian random fields. Consider a real Gaussian field with arbitrary power spectrum $P(k)$ and rms fluctuation $\sigma$. The regions of space for which the field has the value $\nu \sigma$ form a set of two-dimensional surfaces. Ryden [15] defined the quantity $N_{3}(\nu)$ to be the mean area per unit volume of these surfaces. The intersection of this set of surfaces with an arbitrary plane produces a set of curves, with mean length $N_{2}(\nu)$ per unit area of the plane. Finally, we can consider the intersection of this set of surfaces with an arbitrary straight line; the quantity $N_{1}(\nu)$ is the number of intersections of this line (per unit length of the line) with the set of surfaces. Ryden showed that 15

$$
N_{3}(\nu)=\frac{4}{\pi} N_{2}(\nu)=2 N_{1}(\nu)
$$


where

$$
N_{1}(\nu)=\frac{1}{\pi \sqrt{3}}\left\langle k^{2}\right\rangle^{1 / 2} e^{-\nu^{2} / 2},
$$

and the value of $\left\langle k^{2}\right\rangle$ depends on the power spectrum as

$$
\left\langle k^{2}\right\rangle=\frac{\int P(k) k^{4} d k}{\int P(k) k^{2} d k}
$$

These results can be used to derive analytic expressions for the topological defect density in our model. Consider first the area per unit volume, $(A / V)$, for domain walls. In terms of the Ryden notation, it is obvious that

$$
(A / V)=N_{3}(0)=\frac{2}{\pi \sqrt{3}}\left\langle k^{2}\right\rangle^{1 / 2}
$$

(Although we confine our attention to unbiased field configurations, i.e., configurations for which positive and negative values of $\phi_{i}$ are equally likely, so that $\nu=0$, our results can easily be generalized to biased configurations by taking a nonzero value for $\nu$ ). Now consider the case of cosmic strings. The strings can be considered to lie at the intersection of two sets of $\nu=0$ surfaces, corresponding to $\phi_{1}=0$ and $\phi_{2}=0$, respectively. Suppose that we are sitting on a surface corresponding to $\phi_{1}=0$, with area per unit volume $N_{3}(0)$, and we wish to calculate the length per unit area of the intersection of this surface with the $\phi_{2}=0$ surface. Although the $\phi_{1}=0$ surface is highly irregular, if we take a small enough patch, it will be locally flat (assuming the field is smoothed on small scales), so that the length per area of this intersection is just $N_{2}(0)$. Hence, the total length per unit area, $(L / V)$, of cosmic string is just

$$
(L / V)=N_{2}(0) N_{3}(0)=\frac{1}{3 \pi}\left\langle k^{2}\right\rangle
$$

Note that this result agrees exactly with the analytic expression for $(L / V)$ derived by Vishniac et al. [16] using different methods. Finally, we consider the case of monopoles, which are taken to lie at the intersection of the surfaces corresponding to the zeros of three independent Gaussian fields. The intersection of two of these sets of surfaces $\left(\phi_{1}=0\right.$ and $\left.\phi_{2}=0\right)$ defines a set of curves with length per unit volume given by equation (10). While these curves are quite irregular, they are locally straight on small enough scales (assuming the fields are smoothed on small scales), so that their intersection with the third set of surfaces $\left(\phi_{3}=0\right)$ gives $N_{1}(0)$ as the number of intersections per unit length of the curves. Then the monopole density per unit volume, $(N / V)$, is

$$
(N / V)=N_{1}(0) N_{2}(0) N_{3}(0)=\frac{1}{3^{3 / 2} \pi^{2}}\left\langle k^{2}\right\rangle^{3 / 2}
$$

Although our results are applicable to arbitrary power spectra, for definiteness we now restrict our attention to the case $n=0$, which corresponds to uncorrelated fields. This is the most physically relevant case, because a causal phase transition will lead to fields which are uncorrelated on scales larger than the horizon, while the smoothing length can be identified with the scale over which the field tends to be correlated. For $n=0$ with Gaussian smoothing, equations (2), (5), and (8) yield

$$
\left\langle k^{2}\right\rangle=\frac{3}{2 r_{0}^{2}}
$$

Substituting this result into equations (9), (10) and (11), we obtain

$$
\begin{array}{ll}
(A / V)=\frac{\sqrt{2}}{\pi r_{0}} & (\text { walls }), \\
(L / V)=\frac{1}{2 \pi r_{0}^{2}} & (\text { strings }), \\
(N / V)=\frac{1}{2^{3 / 2} \pi^{2} r_{0}^{3}} & \text { (monopoles). }
\end{array}
$$

We now compare these results to numerical simulations. Using the procedure outlined above, we have simulated the formation of domain walls, cosmic strings, and monopoles on a $128^{3}$ lattice, using 1, 2, and 3 independent Gaussian random fields, respectively. For the case of cosmic strings, we use two different cubic lattices, staggered with respect 
to each other by $1 / 2$ of a lattice spacing in the $x, y$, and $z$ directions (i.e., the vertices of one lattice lie at the centers of the cells of the other lattice). We then set down independent Gaussian fields on each lattice, and the strings are taken to lie at the intersection of the zeros of the two fields. For monopoles, we take three different cubic lattices, staggered with respect to each other by $1 / 3$ of a lattice spacing in the $x, y$, and $z$ directions, and the monopoles are taken to lie at the intersection of the three surfaces defined by $\phi_{1}=0, \phi_{2}=0$, and $\phi_{3}=0$.

In each case, we produce 4 different realizations to obtain a mean defect number density and standard deviation. In Fig. 1, we show the domain wall area per unit volume, (A/V), in our simulations as a function of smoothing length $r_{0}$. In Fig. 2 we give the string length per unit volume, $(\mathrm{L} / \mathrm{V})$, as a function of $r_{0}$, and in Fig. 3 we give the number of monopoles per unit volume, $(N / V)$, as a function of $r_{0}$. (Here we make no distinction between monopoles and antimonopoles, although we will do so later). Also in these figures, we show as solid lines the theoretical values for $(A / V),(L / V)$, and $(N / V)$ from equations (13). The disagreement is obvious. Clearly the lattice-based simulations fail to produce the true continuum number densities for topological defects.

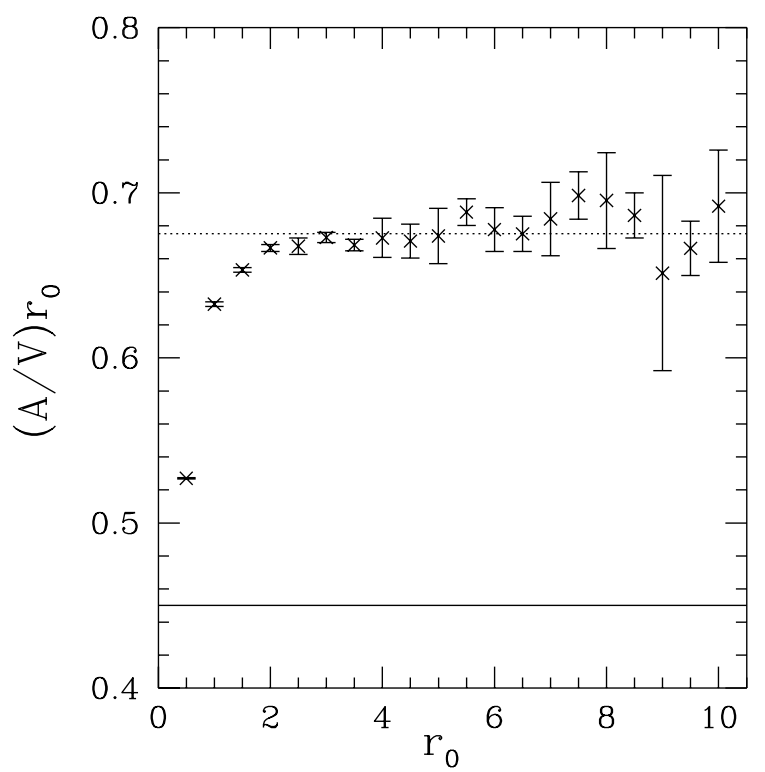

FIG. 1. The total domain wall area per unit volume, $(A / V)$, multiplied by the smoothing length $r_{0}$, as a function of $r_{0}$, for Gaussian smoothing, where $r_{0}$ is measured in units of the lattice spacing. The solid line gives the analytic prediction for $(A / V) r_{0}$ for this model in the continuum limit. The dotted line is the lattice-based analytic prediction. 


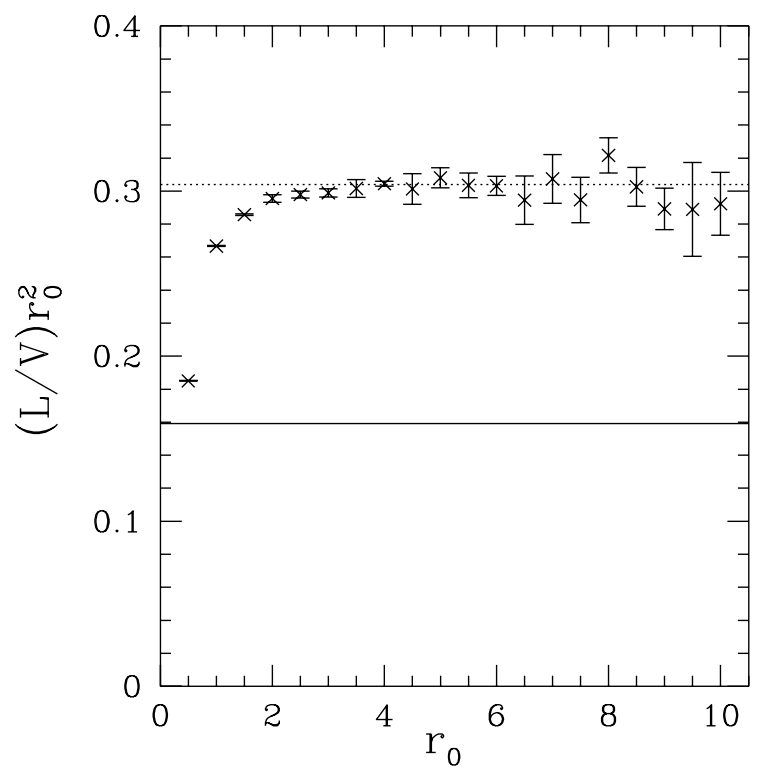

FIG. 2. The total string length per unit volume, $(L / V)$, multiplied by the square of the smoothing length $r_{0}$, as a function of $r_{0}$, for Gaussian smoothing, where $r_{0}$ is measured in units of the lattice spacing. The solid line gives the analytic prediction for $(L / V) r_{0}^{2}$ for this model in the continuum limit. The dotted line is the lattice-based analytic prediction.

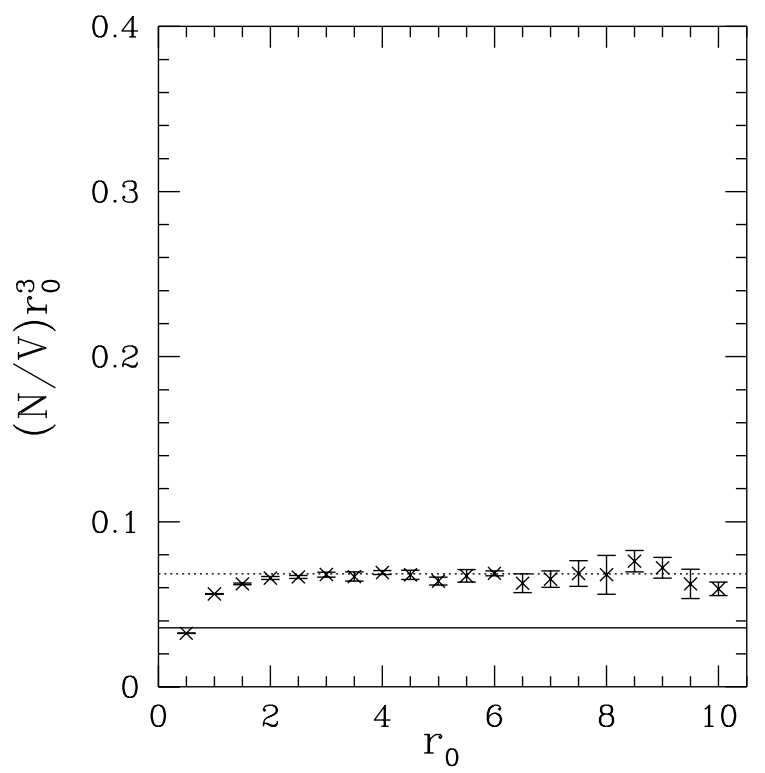

FIG. 3. The total number of monopoles per unit volume, $(N / V)$, multiplied by the cube of the smoothing length $r_{0}$, as a function of $r_{0}$, for Gaussian smoothing, where $r_{0}$ is measured in units of the lattice spacing. The solid line gives the analytic prediction for $(N / V) r_{0}^{3}$ for this model in the continuum limit. The dotted line is the lattice-based analytic prediction. 


\section{THE SOLUTION}

Although our analytic expressions for the topological defect densities given in the previous section fail to agree with the results of our numerical simulations, we can use a different set of arguments to derive "lattice-based" analytic expressions which do agree with the lattice simulations. Consider first the case of domain walls, and consider a single face between two cells on the lattice. This face will be occupied by a domain wall if the cell on one side of it has $\phi>0$ and the other cell has $\phi<0$, or vice versa. This occupation probability, $p_{o c c}$, can be derived from Sheppard's theorem [17] when $\phi$ is a Gaussian field. We obtain

$$
p_{o c c}=\frac{1}{\pi} \cos ^{-1}\left[\frac{\xi(r)}{\xi(0)}\right],
$$

where $r$ is the spacing between the lattice cells, and $\xi$ is the two-point correlation function, which is the Fourier transform of the power spectrum. For an $n=0$ power spectrum smoothed with our Gaussian window function, we have $\xi(r) / \xi(0)=\exp \left(-r^{2} / 4 r_{0}^{2}\right)$, and

$$
p_{o c c}=\frac{1}{\pi} \cos ^{-1}\left[e^{-r^{2} / 4 r_{0}^{2}}\right]
$$

For $r_{0} \gg r$ (i.e., when the smoothing length is large compared to the lattice spacing) this reduces to

$$
p_{o c c}=\frac{1}{\pi \sqrt{2} r_{0}}
$$

This result can be used to derive not only the domain wall density, but the string and monopole densities as well. For the case of domain walls, each cell is bounded by six faces, each of which is shared between two cells, so that the total area per unit volume is $(A / V)=3 p_{o c c}$. In our string simulation, a string forms at the intersection of two different lattice faces, where the two lattices are staggered by half a lattice spacing. For a single cell on one lattice, there are 24 such possible intersections, each with length $r / 2$ (where $r$ is the lattice spacing) and each shared by two cells. Hence, the total length per unit volume is $(L / V)=6 p_{o c c}^{2}$. Finally, for the monopoles, a monopole forms at the intersection of three lattice faces on three different staggered lattices. On a single cell of one lattice, there are 12 possible intersection sites, each of which is shared between two cells. Thus, $(N / V)=6 p_{o c c}^{3}$.

Combining these results with the value for $p_{\text {occ }}$ given in equation (16), we obtain the following values for the topological defect densities:

$$
\begin{aligned}
(A / V)_{\text {lat }} & =\frac{3}{\pi \sqrt{2} r_{0}} & & (\text { walls }), \\
(L / V)_{\text {lat }} & =\frac{3}{\pi^{2} r_{0}^{2}} & & (\text { strings }), \\
(N / V)_{l a t} & =\frac{3}{\pi^{3} \sqrt{2} r_{0}^{3}} & & \text { (monopoles) }
\end{aligned}
$$

where the lat subscript denotes the fact that this analytic calculation is lattice-based, rather than derived from a continuum calculation.

These values for the defect densities are shown in Figs. 1-3 as dotted lines. As $r_{0}$ becomes sufficiently large compared to the lattice spacing, the numerical results converge to a defect density in good agreement with equations (17), although the fluctuations between realizations also increase with $r_{0}$. Clearly, our results in equations (17) give the correct defect densities to compare with our lattice-based simulations.

However, this leaves an embarrassing question: why do our simulations fail to agree with the continuum calculations given in equations (13) and in reference [16]? Even without reference to the numerical simulations, we have two predictions for the defect densities, i.e. equations (13) and (17), which disagree with each other. What is the origin and significance of this discrepancy?

In fact, the problem here is related to a classic geometric fallacy [18]. Consider an isosceles right triangle with sides of unit length. The hypotenuse can be represented as a series of steps (Fig. 4). The length of the hypotenuse, represented in this way, is 2 . As the number of steps $N$ is increased, this length remains 2 , even in the limit where $N \rightarrow \infty$. The fundamental problem is that the hypotenuse is not the $N \rightarrow \infty$ limit of the zigzag curve. Similarly, it is impossible to represent accurately a smooth surface or curve on a cubic lattice. While smoothing increases the length scale of curvature for the smooth surface (or, as in Fig. 4, decreases the effective step size), the lattice representation of the surface never reaches the continuum limit. 

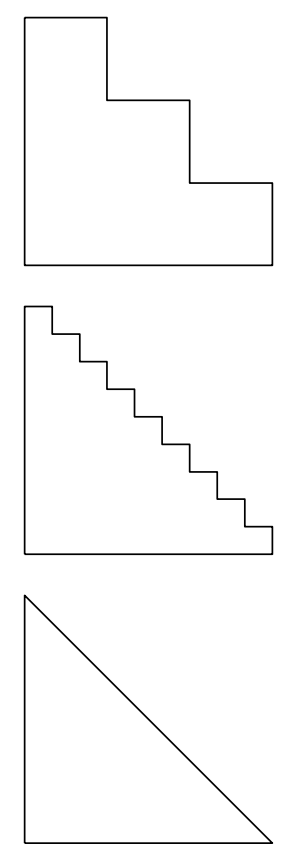

FIG. 4. An illustration of a classic geometric fallacy: as the number of steps goes to infinity, the length of the hypotenuse remains at 2 , rather than approaching the correct value of $2^{1 / 2}$.

Armed with this information, we can now calculate the expected ratio of the continuum value for $(A / V)$ given in equation (13) and the lattice value in equation (17). Suppose that we represent a locally flat surface with area $A$ and normal vector $\mathbf{n}$ on a cubic lattice. The area $A$ then forms a triangular face of a pyramid with sides of length $x, y$, and $z$. The volume of the pyramid is $V=\frac{1}{3} A n=\frac{1}{6} x y z$, so that $A=\frac{1}{2} x y z / n$. The lattice value for this surface area is just $A_{\text {lat }}=\frac{1}{2}(x y+x z+y z)$. Then $(A / V)_{l a t} /(A / V)$ is just the mean value of the ratio of $A_{l a t}$ to $A$, which is

$$
\begin{aligned}
\frac{(A / V)_{\text {lat }}}{(A / V)} & =\left\langle\frac{A_{\text {lat }}}{A}\right\rangle, \\
& =\left\langle\frac{\frac{1}{2}(x y+y z+x z)}{\frac{1}{2} x y z / n}\right\rangle, \\
& =\langle\cos \alpha+\cos \beta+\cos \gamma\rangle,
\end{aligned}
$$

where $\alpha, \beta$, and $\gamma$ are the angles between $\mathbf{n}$ and the $x, y$, and $z$ axes. Since $\mathbf{n}$ is distributed isotropically, we have $\langle\cos \alpha\rangle=\langle\cos \beta\rangle=\langle\cos \gamma\rangle=1 / 2$, and $(A / V)_{\text {lat }} /(A / V)=3 / 2$, which is exactly the value obtained by comparing equation (13) to equation (17).

In fact, the solution to this problem for the case of domain walls was noted by Press et al. [10] and has been used in all domain wall simulations [10] - [12]. In the Press, et al., procedure, the area of a domain wall is simply multiplied by the factor $1 /(|\cos \alpha|+|\cos \beta|+|\cos \gamma|)$, resulting in the correct continuum value for the domain wall area.

A similar weighting procedure cannot be applied in a straightforward way to simulations of cosmic strings and monopoles. For strings, a similar calculation yields $(L / V)_{l a t} /(L / V)=\left\langle(x+y+z) / \sqrt{x^{2}+y^{2}+z^{2}}\right\rangle=\frac{3}{2}$, while the actual ratio from equations (13) and (17) is $(L / V)_{\text {lat }} /(L / V)=6 / \pi=1.91$, which is slightly larger. For monopoles, our geometrical argument fails completely. There is no obvious reason that $(N / V)$ should be any different in a continuum or lattice calculation, since the monopoles are geometrical points. Nonetheless, equations (13) and (17) yield $(N / V)_{l a t} /(N / V)=6 / \pi$, as in the case of strings.

This secondary lattice dependence is a result of the way in which we have simulated the string and monopole formation. Both the strings and monopoles are treated as arising from intersections of the surfaces defined by the zeros of 2 or 3 independent Gaussian fields. Even when smoothed, these surfaces retain a residual jaggedness, as 
we have already emphasized. The intersection of two such jagged surfaces produces spurious additional intersections which would not be present in the continuum limit. As an example, consider the hypotenuse in Fig. 4. When represented as a series of steps, two such lines can have a large number of intersections, even when the two lines are parallel, while in the continuum limit, there would be at most one intersection, and none in the case where the lines were parallel. This secondary effect does not enter into the domain wall calculation, since the domain walls arise from the zeros of a single field.

This problem can be resolved by linearly interpolating the values of each field on the lattice, effectively representing the zeros of the field as polyhedral, rather than cubic, surfaces. We have tested such a procedure for the case of monopoles. We derive an equation for the value of $\phi$ for each of the three fields in each cubic cell by linearly interpolating on the values of each field at the four points $(0,0,0),(1,0,0),(0,1,0)$, and $(0,0,1)$. We then place a monopole in the cell if the $\phi=0$ planes of the three fields intersect inside the cell. The results are shown in Fig. 5 . They agree extremely well with the continuum predictions, despite the fact that our linear interpolation is still an approximation to the true continuum field.

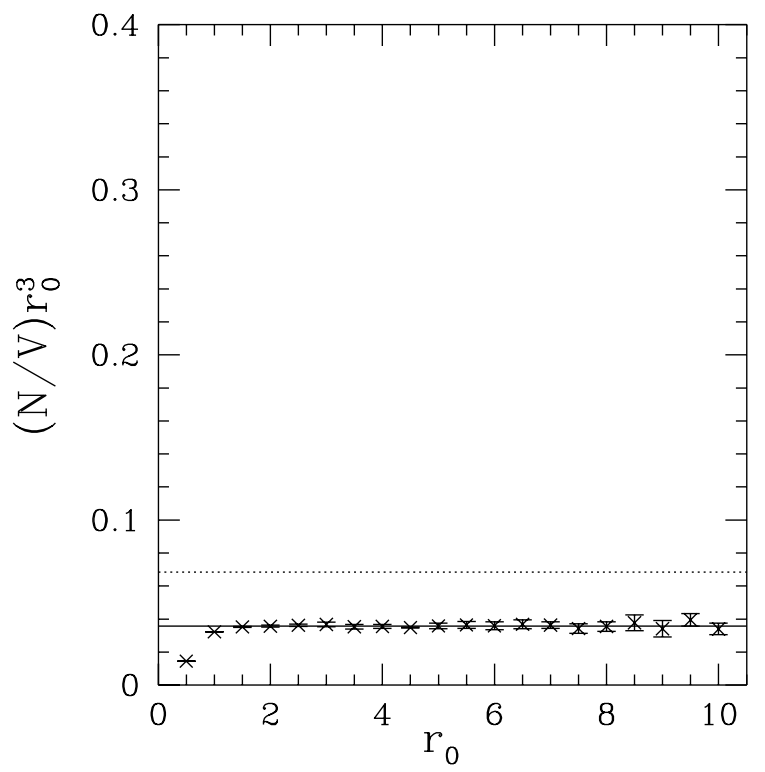

FIG. 5. As in Fig. 3, but with linear interpolation on the three fields used to determine the monopole positions.

This procedure can also be used for cosmic strings. In this case, we take the values of our two fields to lie on the vertices (rather than inside the cells) of the cubic lattice. For each face of the cubic lattice, and for each of the two fields, we use bilinear interpolation 19] on the four values of the field bounding that face to calculate the location of the zeros of the field on that face. This gives the intersection (if any) of the two surfaces $\phi_{1}=0$ and $\phi_{2}=0$ with the given lattice face. If both surfaces do intersect a given lattice face, then we obtain two curves (one for each field) which give the intersection of the $\phi_{1}=0$ and $\phi_{2}=0$ surfaces of that field with the lattice face. The intersection of these two curves gives the point at which a string passes through that face of the cubic lattice. We then connect these intersection points with straight segments (now no longer parallel to the lattice edges) to obtain the string network. (The general expression for the location of a single $\phi=0$ curve on a lattice face, obtained from bilinear interpolation, is $a x y+b x+c y+d=0$, where $a-d$ are constants and the face is taken to lie in the $x-y$ plane. Hence, this procedure can produce two intersections of the $\phi_{1}=0$ and $\phi_{2}=0$ curves on a single lattice face, corresponding to two strings passing through a single face of the lattice. In this case we treat the string as a single small loop with length equal to twice the distance between the intersection points. Such tiny loops become relatively less important as the smoothing length increases). The resulting values for $L / V$ as a function of smoothing length are displayed in Fig. 6. Again, agreement with our continuum predictions is excellent. 


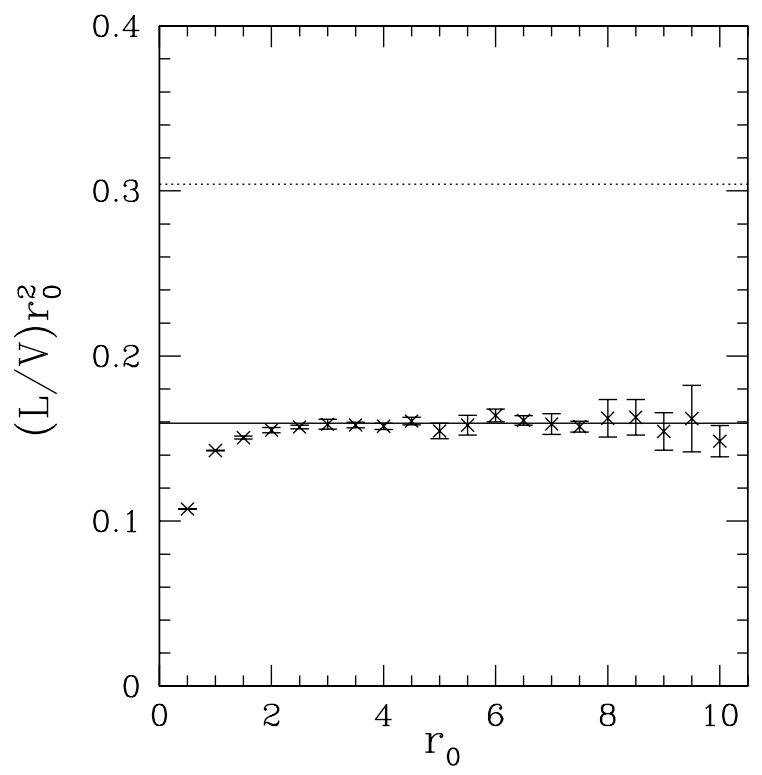

FIG. 6. As in Fig. 2, but with linear interpolation on the two fields used to determine the cosmic string positions.

We can use this linearly-interpolated string network to calculate a "lattice-free" estimate of $f_{\infty}$, the fraction of string length in the form of infinite strings, which we define, as in Ref. [8], to be strings which cross the entire simulation volume in either the $x, y$, or $z$ direction. We have calculated $f_{\infty}$ as a function of smoothing length for our linearly-interpolated string simulations. The results are given in Fig. 7.

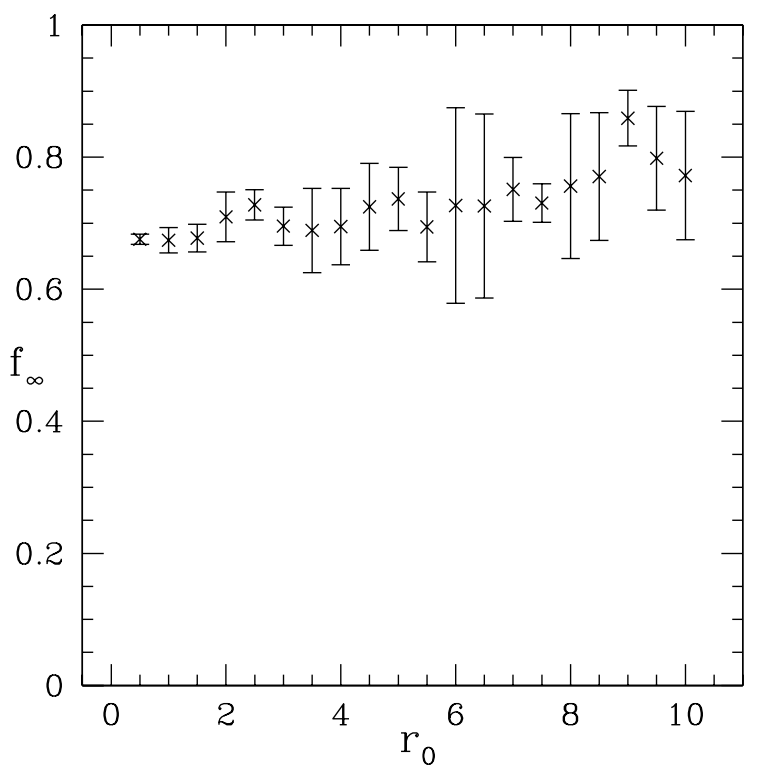

FIG. 7. The fraction of total string length in the form of infinite strings, $f_{\infty}$, as a function of smoothing length, $r_{0}$, for Gaussian smoothing, where linear interpolation on the two fields is used to determine the cosmic string positions.

To obtain an estimate of the lattice-free value of $f_{\infty}$, we want to choose values of $r_{0}$ which are large enough compared to the lattice spacing to eliminate lattice effects, but small enough compared to the total box size to avoid 
large fluctuations from one run to the next. The results shown in Fig. 6 suggest that the range $3 \leq r_{0} \leq 7.5$ should be suitable. When we perform a weighted average of the values of $f_{\infty}$ in this range, we obtain: $f_{\infty}=0.716 \pm 0.015$. This result represents a true lattice-free estimate of $f_{\infty}$, but we do expect it to depend on the actual window function used for smoothing. Our lattice-free results are strikingly similar to earlier results obtained with Gaussian smoothing in a simulation where strings were constrained to lie on a cubic lattice: $f_{\infty}=0.71 \pm 0.01$ [8]. It would appear that $f_{\infty}$, unlike $(L / V)$, is not much affected by the use of a cubic lattice.

For the case of monopoles, one interpretation of our results is that our simple-minded lattice simulation leads to the formation of spurious closely-spaced monopole-antimonopole pairs. To test this hypothesis, we must distinguish between monopoles and antimonopoles in the simulation. Consider first the case where the $\phi_{1}=0$ surface lies in the $y-z$ plane, the $\phi_{2}=0$ surface lies in the $x-z$ plane, and the $\phi_{3}=0$ surface lies in the $x-y$ plane, so that the only non-zero derivatives of the field are $\phi_{1, x}, \phi_{2, y}$ and $\phi_{3, z}$. In this case, we define zeros of the three fields to be monopoles if $\phi_{1, x} \phi_{2, y} \phi_{3, z}>0$, and antimonopoles if this product is negative. For other field configurations, the sign of the product of the derivatives which corresponds to a monopole changes each time we alter the direction of one pair of zeros. For instance, if the $\phi_{1}=0$ surface lies in the $x-z$ plane and the $\phi_{2}=0$ surface likes in the $y-z$ plane, but the $\phi_{3}=0$ surface remains in the $x-z$ plane, then $\phi_{1, y} \phi_{2, x} \phi_{3, z}$ is now negative for monopoles and positive for antimonopoles. Similarly, if we now permute the directions of the zeros of the $\phi_{2}$ and $\phi_{3}$ fields, then $\phi_{1, y} \phi_{2, z} \phi_{3, x}$ is positive for monopoles and negative for antimonopoles.

Using this definition of monopoles and antimonopoles, we have checked the consequences of allowing closelyseparated monopole-antimonopole pairs in our simulation to annihilate. We use the same monopole simulation as in Fig. 3, but now for each monopole in the simulation, we check for the existence of any antimonopoles within an "annihilation distance" $r_{A}$. If an antimonopole is found within this distance, both the monopole and antimonopole are removed from the simulation. Thus, in our final configuration, all remaining monopoles are separated from antimonopoles by a minimum distance of $r_{A}$. We have fixed the smoothing length $r_{0}$ and calculated the monopole+antimonopole number density $(N / V)$ as a function of the annihilation distance $r_{A}$. The results are shown in Figs. 8 and 9 for smoothing lengths of 6 and 8 lattice spacings, respectively. The monopole density $(N / V) r_{0}^{3}$ decreases as a function of $r_{A}$, and it must obviously go to zero as $r_{A}$ reaches the size of the simulation volume. However, it appears that as $r_{A}$ approaches $r_{0}$, a plateau value for $(N / V) r_{0}^{3}$ is reached. Moreover, the plateau value of $(N / V) r_{0}^{3}$ lies at the continuum prediction of equation (13). The statistics are rather poor, but our results do suggest that one interpetation of the spurious monopoles in the lattice simulations is that such monopoles are produced as closely-spaced monopole-antimonopole pairs.

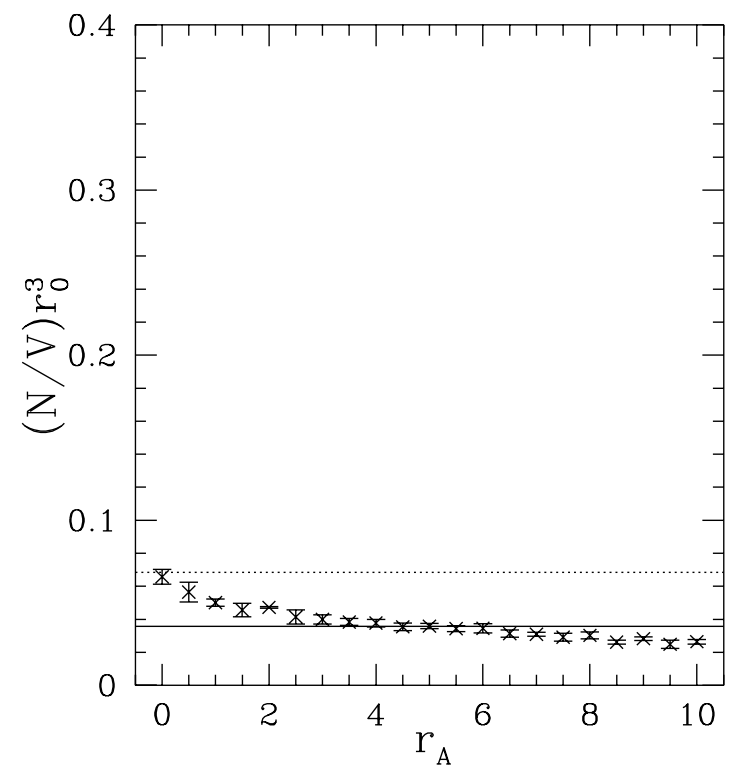


FIG. 8. The total number of monopoles+antimonopoles per unit volume, $(N / V)$, multiplied by the cube of the smoothing length $r_{0}$, as a function of the "annihilation distance" $r_{A}$, where monopole-antimonopole pairs separated by a distance less than $r_{A}$ have been removed from the simulation, and $r_{0}$ is fixed at 6 lattice spacings. The solid line gives the analytic prediction for $(N / V) r_{0}^{3}$ in the continuum limit. The dotted line is the lattice-based analytic prediction.

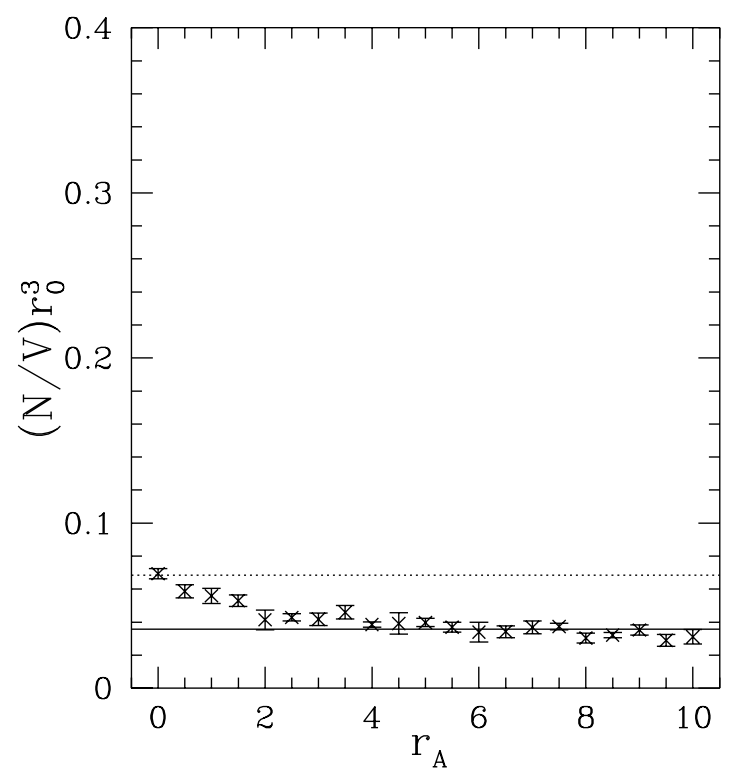

FIG. 9. As in Fig. 8, with $r_{0}=8$ lattice spacings.

\section{DISCUSSION}

Our results indicate that lattice simulations for the formation of topological defects must be used with some caution. Even when the Gaussian fields used in these simulations are smoothed to reduce lattice effects on small scales, a residual lattice dependence remains. Another way to express this result is to note that smoothing eliminates the small-scale inhomogeneity introduced by using a lattice, but it cannot eliminate the anisotropy. Strings, for example, simulated on a cubic lattice are required to lie only in the $x, y$, or $z$ directions, while a genuine continuum simulation would allow an arbitrary direction for the string segments.

For the case of domain walls, a simple multiplicative factor which depends on the direction of the domain wall can be used to eliminate this problem; all domain wall simulations have used such a factor [10] - 12. For strings, the correct multiplicative factor does not amount to simply averaging over the directions for a straight string: in the lattice simulations there is residual small-scale structure along the strings and probably an accompanying distribution of tiny loops (of order the lattice spacing). These effects change the multiplicative factor from 1.5 (its value when only anisotropy is taken into account) to 1.91, but they should not affect the length distribution of loops (except for the smallest loops). In practice, nearly all previous studies of cosmic strings have used a simple lattice simulation of the kind described here [2] - [8], as have some monopole simulations [13] (although see Ref. [14] for a continuum field treatment of monopoles).

For cosmic strings and monopoles, the problem can be solved by linearly interpolating the values of the field on the lattice. This procedure yields the correct continuum value for the monopole and string densities; for the case of monopoles, a similar result can be achieved in a simple-minded lattice simulation by eliminating close pairs of monopoles. For the case of cosmic strings, this interpolation scheme produces a truly lattice-free estimate of the total string length in the form of infinite strings: $f_{\infty}=0.716 \pm 0.015$. This is nearly identical to the value obtained from a simple lattice simulation (with Gaussian smoothing) where the strings were constrained to lie on the edges of the lattice [3]. Apparently $f_{\infty}$ is much less sensitive to lattice effects than is the total string density. This is not surprising, since the effect of the cubic lattice is to multiply the string density by a geometric factor; if this factor is the same 
for both closed loops and infinite strings, then $f_{\infty}$ is unaffected. However, even in these lattice-free simulations, we expect the value of $f_{\infty}$ to depend on the window function used for smoothing (as in reference [8]).

This linear interpolation procedure can also be extended to domain walls, providing an alternative to the weighting factor used in references [10] - [12]. In practice, these two methods should give nearly identical results for any domain wall quantities of interest.

\section{ACKNOWLEDGMENTS}

We thank U.-L. Pen for numerous helpful suggestions, including the linear interpolation scheme for the lattice-free string simulations. We are grateful to S. Larsson for helpful comments on the manuscript. R.J.S. is supported by the Department of Energy (DE-FG02-91ER40690) and by NASA (NAG 5-3111) at Ohio State, and by the Department of Energy and NASA (NAG 5-2788) at Fermilab. A.V. is supported by the National Science Foundation.

[1] A. Vilenkin and E.P.S. Shellard, Cosmic Strings and other Topological Defects, Cambridge University Press, Cambridge (1994); M.B. Hindmarsh and T.W.B. Kibble, Rep. Prog. Phys. 55, 478 (1995).

[2] T. Vachaspati and A. Vilenkin, Phys. Rev. D 30, 2036 (1984).

[3] R.J. Scherrer and J. Frieman, Phys. Rev. D 33, 3556 (1986).

[4] T. Vachaspati, Phys. Rev. D 44, 3723 (1991).

[5] A. Yates and T.W.B. Kibble, Phys. Lett. B 364, 149 (1995).

[6] M. Hindmarsh and K. Strobl, Nucl. Phys. B 437, 471 (1995).

[7] J. Robinson and A. Yates, Phys. Rev. D 54, 5211 (1996).

[8] R.J. Scherrer and A. Vilenkin, Phys. Rev. D 56, 647 (1997).

[9] J. Borrill, Phys. Rev. Lett. 76, 3255 (1996).

[10] W.H. Press, B.S. Ryden, and D.N. Spergel, Astrophys. J. 347, 590 (1989).

[11] D. Coulson, Z. Lalak and B. Ovrut, Phys. Rev. D 53, 4237 (1996).

[12] S.E. Larsson, S. Sarkar, and P.L. White, Phys. Rev. D 55, 5129 (1997).

[13] E. Copeland, D. Haws, T.W.B. Kibble, D. Mitchell, and N. Turok, Nucl. Phys. B 298, 445 (1988).

[14] D.P. Bennett and S.H. Rhie, Phys. Rev. Lett. 65, 1709 (1990).

[15] B.S. Ryden, Ap.J. Lett. 333, L41 (1988); B.S. Ryden, Pub. Astr. Soc. Pac. 100, 1360 (1988).

[16] E. T. Vishniac, K. A. Olive, and D. Seckel, Nucl. Phys. B 289, 717 (1987).

[17] A.J.S. Hamilton, J.R. Gott, and D. Weinberg, Ap.J. 309, 1 (1986).

[18] H.E. Dudeney, The Canterbury Puzzles (1919).

[19] W.H. Press, B.P. Flannery, S.A. Teukolsky, and W.T. Vetterling, Numerical Recipes, Cambridge University Press, Cambridge (1986). 